\title{
Bioanalysis
}

\section{White Paper on Recent Issues in Bioanalysis: 'A global bioanalytical community perspective on last decade of incurred samples reanalysis (ISR)' (Part 1 - small molecule regulated bioanalysis, small molecule biomarkers, peptides \& oligonucleotide bioanalysis)}

The $201812^{\text {th }}$ Workshop on Recent Issues in Bioanalysis (12th WRIB) took place in Philadelphia, PA, USA on April 9-13, 2018 with an attendance of over 900 representatives from pharmaceutical/biopharmaceutical companies, biotechnology companies, contract research organizations and regulatory agencies worldwide. WRIB was once again a 5-day full immersion in bioanalysis, biomarkers and immunogenicity. As usual, it was specifically designed to facilitate sharing, reviewing, discussing and agreeing on approaches to address the most current issues of interest including both small- and largemolecule bioanalysis involving LC-MS, hybrid ligand binding assay (LBA)/LC-MS and LBA/cell-based assays approaches. This 2018 White Paper encompasses recommendations emerging from the extensive discussions held during the workshop, and is aimed to provide the bioanalytical community with key information and practical solutions on topics and issues addressed, in an effort to enable advances in scientific excellence, improved quality and better regulatory compliance. Due to its length, the 2018 edition of this comprehensive White Paper has been divided into three parts for editorial reasons. This publication (Part 1) covers the recommendations for LC-MS for small molecules, peptides, oligonucleotides and small molecule biomarkers. Part 2 (hybrid LBA/LC-MS for biotherapeutics and regulatory agencies' inputs) and Part 3 (large molecule bioanalysis, biomarkers and immunogenicity using LBA and cell-based assays) are published in volume 10 of Bioanalysis, issues 23 and 24 (2018), respectively.

First draft submitted: 26 September 2018; Accepted for publication: 10 October 2018; Published online: 29 November 2018

Index

Introduction

Discussion topics

- Small molecule regulated bioanalysis \& peptides by LCMS

- Small molecule biomarkers by LCMS

- Oligonucleotides

Discussions, consensus \& conclusions

- Small molecule regulated bioanalysis \& peptides by LCMS

- Small molecule biomarkers by LCMS

- Oligonucleotides

Recommendations

- Small molecule regulated bioanalysis \& peptides by LCMS

- Small molecule biomarkers by LCMS

- Oligonucleotides

References

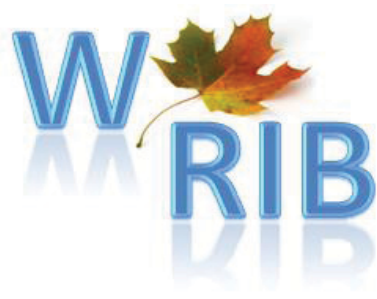

Jan Welink ${ }^{1}$, Yuanxin $\mathrm{Xu}^{2}$, Eric Yang $^{3}$, Amanda Wilson ${ }^{4}$, Neil Henderson $^{5}$, Lina Luo ${ }^{6}$, Stephanie Fraser ${ }^{6}$, Uma Kavita7, Adrien Musuku ${ }^{8}$, Christopher James ${ }^{9}$, Daniela Fraier $^{10}$, Yan Zhang ${ }^{7}$, Dina Goykhman ${ }^{11}$, Scott Summerfield ${ }^{12}$, Eric Woolf ${ }^{11}$, Tom Verhaeghe ${ }^{13}$, Nicola Hughes $^{14}$, Alexander Behling ${ }^{15}$, Kirk Brown ${ }^{2}$, Alex Bulychev $^{16}$, Michael Buonarati ${ }^{17}$, Elana Cherry ${ }^{18}$, Seongeun (Julia) Cho ${ }^{19}$, Isabelle Cludts ${ }^{20}$, Lieve Dillen ${ }^{13}$, Robert Dodge 21, Anna Edmison ${ }^{18}$, Fabio Garofolo*,22, Rachel Green ${ }^{23}$, Sam Haidar ${ }^{19}$, Charles Hottenstein ${ }^{3}$, Akiko Ishii-Watabe ${ }^{24}$, Hyun Gyung Jang $^{25}$, Allena Ji ${ }^{26}$, Barry Jones $^{27}$, Sean Kassim ${ }^{19}$, Mark $\mathrm{Ma}^{28}$, Meena ${ }^{29}$, Gustavo Mendes Lima Santos ${ }^{30}$, Daniel A Norris $^{31}$, Tate Owen ${ }^{32}$, Steven Piccoli33, Ragu Ramanathan ${ }^{6}$, Ingo Röhl ${ }^{34}$, Anton I Rosenbaum ${ }^{35}$, Yoshiro Saito $^{24}$, Timothy Sangster ${ }^{36}$, Natasha Savoie ${ }^{37}$, Christopher Stebbins $^{38}$, Jens Sydor ${ }^{39}$, Nico van de Merbel $^{40}$, Daniela Verthelyi ${ }^{19}$, Stephen Vinter ${ }^{41}$ \& Emma Whale ${ }^{41}$

\footnotetext{
*Author for correspondence:

f.garofolo@angelini.it
}

Author affiliations and Regulatory Agencies Disclaimer can be found at the end of this article 


\section{Key terms}

Biomarker qualification: A conclusion based on a formal regulatory process, that within the stated context of use, a medical product development tool can be relied upon to have a specific interpretation and application in medical product development and regulatory review [65].

Context of use: A statement that fully and clearly describes the way the medical product development tool is to be used and the medical product development-related purpose of the use [65].

\section{Acronyms}

\begin{tabular}{|c|c|}
\hline ADA: & Anti-drug antibody \\
\hline ADME: & Absorption, distribution, metabolism, and excretion \\
\hline AEX-HPLC: & Anion exchange high performance liquid chromatography \\
\hline API: & Active pharmaceutical ingredient \\
\hline ASO: & Antisense oligonucleotides \\
\hline BAV: & Biomarker assay validation \\
\hline bDNA: & Branched DNA \\
\hline BE: & Bioequivalence \\
\hline BMV: & Bioanalytical method validation \\
\hline C-Path: & Critical Path Institute \\
\hline CDCA: & Chenodeoxycholic acid \\
\hline CRO: & Contract research organization \\
\hline DDI: & Drug-drug interaction \\
\hline DMPK: & Drug metabolism and pharmacokinetics \\
\hline ELISA: & Enzyme-linked immunosorbent assay \\
\hline FFP: & Fit for purpose \\
\hline FIH: & First in human \\
\hline GCA: & Glycocholic acid \\
\hline GCDCA: & Glycochenodeoxycholic acid \\
\hline GCC: & Global CRO Council \\
\hline GLP: & Good Laboratory Practices \\
\hline GTMP: & Gene therapy medicinal product \\
\hline HPLC-FL: & High performance liquid chromatography-fluorescence detection \\
\hline HRMS: & High resolution mass spectrometry \\
\hline IA: & Immunoaffinity \\
\hline IBA: & Individual bile acids \\
\hline ICD: & Informed consent document \\
\hline IS: & Internal standard \\
\hline ISR: & Incurred sample reproducibility \\
\hline ISS: & Incurred sample stability \\
\hline LBA: & Ligand binding assay \\
\hline LCMS: & Liquid chromatography mass spectrometry \\
\hline LLOQ: & Lower limit of quantitation \\
\hline LNP: & Lipid nanoparticle \\
\hline M\&S: & Modeling and simulation \\
\hline miRNA: & MicroRNA \\
\hline MIST: & Metabolites in safety testing \\
\hline MRM: & Multiple-reaction monitoring \\
\hline
\end{tabular}




\begin{tabular}{|ll|}
\hline MS: & Mass spectrometry \\
\hline NAb: & Neutralizing antibody \\
\hline NCE: & New chemical entity \\
\hline PBPK: & Physiologically based pharmacokinetic \\
\hline PCR: & Polymerase chain reaction \\
\hline PD: & Pharmacodynamics \\
\hline PEG: & Polyethylene glycol \\
\hline PK: & Pharmacokinetics \\
\hline PNA: & Peptide nucleic acid \\
\hline PTM: & Post-translational modification \\
\hline QC: & Quality control \\
\hline qPCR or RT-qPCR: & Quantitative PCR or quantitative reverse transcriptase polymerase chain reaction \\
\hline SgRNA: & Single guide RNA \\
\hline siRNA: & Small interfering RNA \\
\hline SIL-IS: & Stable isotope label internal standard \\
\hline SOP: & Standard operating procedure \\
\hline SPE: & Solid-phase extraction \\
\hline TCDCA: & Taurochenoxycholic acid \\
\hline WRIB: & Workshop on Recent Issues in Bioanalysis \\
\hline
\end{tabular}

The $12^{\text {th }}$ edition of the Workshop on Recent Issues in Bioanalysis (12 $2^{\text {th }}$ WRIB) was held in Philadelphia, PA, USA on April 9-13, 2018 with an exceptionally high attendance of over 900 representatives (likely facilitated by the Philadelphia location) from pharmaceutical/biopharmaceutical companies, biotechnology companies, contract research organizations, and regulatory agencies worldwide. The workshop included three sequential main workshop days, two additional advanced specialized sessions and four full-day training sessions that together spanned an entire week in order to allow exhaustive and thorough coverage of all major issues in bioanalysis, biomarkers, and immunogenicity.

As in previous years, this year's WRIB continued to gather a diverse group of international opinion leaders from industry and regulatory authority experts working on both small and large molecules to facilitate sharing and discussions focused on improving quality, increasing regulatory compliance and achieving scientific excellence on bioanalytical issues.

The active contributing chairs included Dr Chris Beaver (Syneos Health), Dr Seongeun (Julia) Cho (US FDA), Dr John Kamerud (Pfizer), Dr Steve Keller (AbbVie), Dr Fabio Garofolo (Angelini Pharma), Dr Joe Marini (Janssen), Dr Adrien Musuku (Pharmascience), Dr Hendrik Neubert (Pfizer), Dr Timothy Olah (Bristol-Myers Squibb), Dr Renuka Pillutla (Bristol-Myers Squibb), Dr Sue Richards (Sanofi), Dr Lauren Stevenson (Biogen), Dr Albert Torri (Regeneron), Mr Stephen Vinter (UK MHRA), Dr Jan Welink (EU EMA), Dr Yuanxin Xu (Alnylam) and Dr Eric Yang (GlaxoSmthKline).

The participation of regulatory agency representatives continued to grow at WRIB [1-18], covering topics and discussions in bioanalysis, biomarkers and immunogenicity:

- Regulated Bioanalysis: Dr Sean Kassim (US FDA), Dr Sam Haidar (US FDA), Dr Seongeun (Julia) Cho (US FDA), Dr Sarah Rogstad (US FDA), Dr Jan Welink (EU EMA), Mr Stephen Vinter (UK MHRA), Ms Emma Whale (UK MHRA), Mr Gustavo Mendes Lima Santos (Brazil ANVISA), Dr Anna Edmison (Health Canada);

- Biomarkers: Dr Christopher Leptak (US FDA), Dr Shashi Amur (US FDA), Dr Shirley Hopper (UK MHRA), Dr Yoshiro Saito (Japan MHLW-NIHS);

- Immunogenicity: Dr Daniela Verthelyi (US FDA), Dr Yow-Ming Wang (US FDA), Dr João PedrasVasconcelos (US FDA), Dr Brian Janelsins (US FDA), Dr Haoheng Yan (US FDA), Dr Meenu Wadhwa (UK MHRA-NIBSC), Dr Isabelle Cludts (UK MHRA-NIBSC), Dr Pekka Kurki (Finland Fimea), Dr Elana Cherry (Health Canada), Dr Akiko Ishii-Watabe (Japan MHLW-NIHS). 
As usual, the $12^{\text {th }}$ WRIB was designed to cover a wide range of topics in bioanalysis, biomarkers and immunogenicity suggested by members of the community, and included daily working dinners and lectures from both industry experts and regulatory representatives, which culminated in open panel discussions amongst the presenters, regulators and attendees in order to reach consensus on items presented in this White Paper.

At this year's WRIB, 39 recent issues ('hot' topics) were addressed and distilled into a series of relevant recommendations. Presented in the current White Paper is the background on each issue, exchanges, consensus and resulting recommendations on these 39 topics, which are separated into the following areas:

\section{LCMS:}

- Small molecule bioanalysis and peptides by LCMS (five topics);

- Small molecule biomarkers by LCMS (four topics);

- Oligonucleotides (five topics).

Hybrid LBA/LCMS:

- Intact biotherapeutic quantification and PK assays (three topics);

- Immunogenicity assays (four topics);

- Biomarker assays (three topics).

LBA and cell-based assays:

- Gene therapy and critical reagents (two topics);

- Biomarker assay validation (three topics);

- Flow cytometry (four topics);

- ADA assays and PK, PD, ADA data correlation (four topics);

- Cut points (two topics).

Key inputs from regulatory agencies:

- In addition to the recommendations on the aforementioned topics, there is a section in the White Paper that focuses specifically on several key inputs from regulatory agencies.

Due to its length, the 2018 edition of this comprehensive White Paper has been divided into three parts for editorial reasons. This publication (Part 1) covers the recommendations for small molecules, peptides, and small molecule biomarkers by LCMS and oligonucleotides. Part 2 (PK, PD \& ADA assays by hybrid LBA/LCMS and regulatory agencies' inputs) and Part 3 (LBA/cell-based assays: immunogenicity, biomarkers and PK assays) are published in volume 10 of Bioanalysis, issues 23 and 24 (2018), respectively.

\section{Discussion topics}

The topics detailed below were considered as the most relevant 'hot topics' based on feedback collected from the $11^{\text {th }}$ WRIB attendees. They were reviewed and consolidated by globally recognized opinion leaders before being submitted for discussion during the $12^{\text {th }}$ WRIB. The background on each issue, discussions, consensus and conclusions are in the next section and a summary of the key recommendations is provided in the final section of this manuscript.

Small molecule regulated bioanalysis \& peptides by LCMS

What ISR/ISS is telling the global bioanalytical community about its methods/procedures

What was the most important value of ISR/ISS in the last decade? How did this help the bioanalytical community to improve bioanalytical method quality and performance? What have we actually learned about our bioanalytical methods from ISR/ISS? Are the bioanalytical methods really better (more rugged, reliable, robust, etc.) than before the ISR requirement? What were the reasons for the major ISR failures in the last 10 years (e.g., metabolite instability)? Are we now able to avoid them in the future? It seems that the number of ISR failures is low. Is ISR still considered necessary? If yes, what are future opportunities for ISR/ISS? Where will the next decade lead? In case of failing ISR for a pivotal study (e.g., BE), will a root cause investigation only be 'a nice to know tool' in 
understanding the problem or may this also be used as a justification to save the study? What would be considered an acceptable justification? In what situations should ISS be performed? Is the use of ISS well understood by the industry based on the recommendations of the 2012 and 2015 White Papers on ISS [5,10]? The US FDA 2013 draft guidance [19] states that ISR should be performed on 7\% of incurred samples in the study regardless of size and the approach in the EMA and Japan MHLW Guidelines [20,21] is based on using 10\% of the first 1000 samples and $5 \%$ of the number of samples exceeding the 1000 samples. Can we come to a consensus?

\section{Revisiting MIST (safety testing of drug metabolites)}

Are the MIST guidelines [22] still appropriate and working well? Will the MIST guidelines remain relevant considering the advancement of analytical technologies for both metabolite profiling and bioanalysis and novel drug entities (e.g., oligonucleotides)? Can MIST approaches be simplified and reduced based on current industry and regulator experience since most small molecule drugs generate multiple metabolites and early deployment of validated methods for multiple metabolites is impractical? What are the strategies combining metabolite profiling and quantitative bioanalysis and the pros and cons of using exploratory methods in GLP safety studies?

\section{Therapeutic peptides - robust LCMS/HRMS \& hybrid LBA/LCMS methods}

What are the most current industry standards for HRMS in regulated bioanalysis of therapeutic peptides versus what was previously recommended in the 2015-2017 White Papers $[10,13,16]$ ? The topics of interest include mass selection window, validation of bioanalytical assays using HRMS instruments versus traditional triple quadrupole, documentation of the processing methods for HRMS platforms, and overall HRMS assay performance? Are there new specific cases where HRMS showed to be more advantageous over standard triple quadrupole MRM workflows versus what was previously discussed in the 2015-2017 White Papers [10,13,16]? Is it common practice to sum MRM to improve assay sensitivity for analysis of therapeutic peptides? What are the benefits, if any, versus the risks? What is the current method of choice for peptides when extra sensitivity and selectivity are needed: reagent-free LCMS or hybrid LBA/LCMS? IA purification is mainly used for large molecules. Is IA with LCMS also needed for peptides? Is reagent-free LCMS enough for peptides? Are there new data to support an evolution of the 2017 White Paper Part 1 [16] recommendations on peptide bioanalysis? If hybrid LBA/LCMS methods are used for peptides, what are the current industry considerations for the selection of the IA capturing antibody? Are there any advantages using less specific generic antibodies? Would the same rules apply to IAP? What about SISCAPA?

\section{Sophisticated bioanalytical methods that drive the quality of PKPD \& PBPK modeling}

While the quality of bioanalytical data is crucial, it is often overlooked when building PBPK or PKPD models. How does FFP bioanalysis apply in the support of building models? What role should bioanalytical experts play in building these models and what should be expected from them?

Next wave of automation \& next wave of issues to overcome: impact on regulated bioanalysis, \& pros/cons of emerging automation in a regulated environment

What level of automation is in use in an industry represented by $70-80 \%$ of innovators and CROs? Does it span the scope of none to end-to-end or is there a focused level that most innovators and CROs are utilizing? What is a reasonable expectation of where automation in the regulated environment can advance in the next 3-5 years? What are the pros/cons of implementing automation in a regulated environment? How is the next wave of automation different from the current one (e.g., task-based, modular or end-to-end)? Should it be expected that the natural progression of automation advances in the discovery space will translate to regulated bioanalysis?

\section{Small molecule biomarkers by LCMS}

Biomarker assay validation for small molecule biomarkers by LCMS: alignment between the recommendations of the C-Path White Paper \& the 2016/2017 White Papers in Bioanalysis (Part 1)

The C-Path White Paper [23] is mainly focused on BAV for LBA. However, small molecule biomarkers, which are typically quantified using LCMS, are very important as surrogates for disease diagnosis, progression, and prognosis and it may be important to add specific guidelines to the C-Path White Paper. Should small molecule BAV be included in the C-Path White Paper? If yes, which of the following tests/parameters should be included: surrogate matrix and surrogate analyte experiments; matrix effects/factor and ion suppression/enhancement; LCMS multiplexing using multiple transitions; criteria to define LLOQ and QCs levels in the calibration curve; selectivity 
and specificity experiments; inclusion of chemical treatments like hydrolysis or derivatization? Should total allowable error be considered for small molecule biomarkers by LCMS? If not, why? Parallelism experiments for small molecule biomarkers by LCMS are different if compared to large molecule biomarkers by LBA. How should this difference be captured in the C-Path White Paper to avoid possible confusion in the parallelism definition between LCMS and LBA? When using a surrogate matrix approach, should endogenous dilution linearity experiments be performed in method validation or is slope comparison sufficient due to availability of reliable reference compounds for small molecule biomarkers? Should this be performed using matrix from multiple individuals? If demonstration of endogenous dilution linearity is important, what does this mean for surrogate analyte methods? For small molecule biomarkers, it is possible to have reliable/authentic reference standards. Hence, are all BMV guidance/guideline experiments appropriate for small molecule BAV? What is the number of validation batches needed for small molecule BAV: LCMS (3) versus LBA (6)? Recent dialogue has suggested that longitudinal QCs are favored over ISR for small molecule biomarkers. Is there a need for ISR at all for small molecule biomarkers? Should co-admin stability (e.g., stability of biomarker in presence of drug) be performed?

\section{Boosting small molecule biomarker assay sensitivity}

When using the authentic matrix method, what is a feasible assay LLOQ when the standard addition method is used for standard curve preparation? Should multiple authentic matrix lots be screened first, and then the lot with the lowest endogenous analyte concentration used for standard curve matrix? Is this the industry standard? Is reducing relative matrix effects the most important parameter to be evaluated besides slope parallelism when using surrogate matrix for standard curve preparation? If yes, why? How is the test for biomarkers assays performed? Is it important to check IS consistency between authentic sample matrix and surrogate matrix? Should there be limits on IS variability for small molecule BAV?

\section{Recent developments in the quantification of individual bile acids as biomarkers for drug induced liver injury}

What are the best/current method development strategies to overcome the high variability of biomarker bile acids and optimize method selectivity and sensitivity? Are there other available options beyond triple quadrupoles used by the industry to achieve better selectivity and sensitivity with bile acids? Is accurate mass measurement a good approach to perform quantification and identification of bile acids simultaneously? How is the issue of endogenous bile acid levels addressed based on biomarker applications (e.g., bile acids as biomarkers for liver injury/liver diseases and bile acids as liver transporter biomarkers)? During method development and validation of the surrogate matrix approach for bile acid analysis, how is the use of a different species matrix (e.g., charcoal stripped dog plasma) as an alternative to measurements in human plasma samples supported? Is a successful parallelism experiment enough? If yes, why? What if parallelism fails?

\section{Endogenous small molecule biomarkers for renal \& hepatic transporters}

A significant number of portfolio compounds trigger DDI risk cutoffs inclusive of the high false-positive rates ( $\sim 30 \%)$ from in-vitro-based transporter DDI evaluations. The use of endogenous biomarkers potentially will allow to stage-gate or at least minimize the needed dose of probe drugs in clinical trials. What are the main industry and regulatory concerns in the use of endogenous biomarkers for supporting or refuting in-vitro DDI predictions? If parallel studies are conducted to demonstrate that endogenous biomarkers give the same DDI risk projections as dosing probe drugs, then would there be any reservations to reduce or completely eliminate the studies which involve dosing of the probe drugs? What are the pros and cons of using endogenous biomarker measurements in FIH studies in order to minimize targeted DDI studies? With appropriate language/permissions in the ICDs and/or clinical protocols, are there any concerns with regard to using left-over PK samples (within appropriate storage, etc.) for endogenous biomarker profiling or DDI projections? Should parallelism tests, using authentic versus surrogate matrix or surrogate analyte, remain a part of the endogenous biomarker qualification/validation plans? How important is it to have SIL-IS for each endogenous biomarker? Is it possible that structural analogues could serve the same purpose? Would there be any concerns with using multiplexed HRMS assays for profiling multiple endogenous biomarkers or would the options to retrospective interrogation of raw data for newly discovered biomarkers (provided adequate language in the ICDs) remain the same? 


\section{Oligonucleotides \\ Recent advances, future trends \& impact on oligonucleotide bioanalysis}

Are the bioanalytical strategies for siRNA, aptamers, oligonucleotides, and conjugated oligonucleotides "one size fits all"? Multiple methods are required for adequate characterization of the PK of therapeutic oligonucleotides, such as ASO. What is the proper bioanalytical method for PK assessment? Is the quantification of vector/vehicle necessary? How important is it to develop PK assays for excipients? Will an assay for the oligonucleotide API suffice? Regarding the issue of the use and validation of formulated versus unformulated standard curves and QCs, will the former suffice for both standards and QCs if it is shown during method development that the denaturation conditions allow for a complete release of the API from the formulated material? Since mRNA can be affected by exposure to extracellular nucleases, what are the best practices for generation of standards and QC samples? Do the same principles of the MIST guidance [22] apply for oligonucleotides (e.g., oligonucleotides metabolite identification and bioanalysis during pre-clinical and clinical development)?

\section{The bioanalytical challenge of oligonucleotide biodistribution: current industry approaches for biological fluids/tissues, unsolved issues, unanswered questions \& future perspectives}

When novel delivery systems are employed for the delivery of nucleotide drugs (such as LNP), what are the regulatory expectations for understanding biodistribution of the formulation? What can we measure for LNP component analysis and distribution? What are the critical characteristics to cover in method qualification for quantitative nucleotide drug measurement in tissue homogenates? What are the best practice approaches for preparation of tissue homogenates for the measurement of nucleotide drugs? How can we improve and facilitate extraction of lipid-containing oligonucleotides especially from tissue? What is the best way of correlating serum/plasma levels of $\mathrm{PK}$ analytes with tissue levels? What is the potential bias encountered when measuring tissue concentrations from biopsies?

\section{Challenges with determination of oligonucleotide immunogenicity}

Given the intracellular site of action of ASOs, what should potential immunogenicity assessment look like? Since any ADA bound to drug would prevent it from reaching its target, should all ADA+ be considered neutralizing? How do we define immunogenicity for complex drugs such as oligonucleotide carrying lipid nanoparticles? How to detect and report pre-treatment immunogenicity? What is the standard definition of immunogenicity? Should the focus continue to be on anti-drug antibodies - that is, monitoring antibody responses for safety and efficacy? Do we need to revise for oligonucleotides? With mRNA/gene therapy that results in expression of previously absent or low abundance proteins, what immunogenicity assessments are necessary with regard to the newly/more highly expressed gene product? Is it necessary to develop an anti-siRNA ADA assay when a short half-life is demonstrated? Is there a cut-off for the half-life that can be prescribed based on the time that it takes for the immune system to mount an antibody response? What are the sensitivity and drug tolerance requirements for the immunogenicity assays for oligonucleotides? What is the relevance of current sensitivity requirement of $100-250 \mathrm{ng} / \mathrm{ml}$ ?

Branched DNA \& RT-qPCR/stem-loop qPCR assays: an in depth understanding of method development \& validation challenges in the quantification of oligonucleotides

PCR methodologies are often not readily available in traditional regulated bioanalysis laboratories. Should the PCR platform become a standard tool in a regulated bioanalysis laboratory for oligonucleotides? What do regulators consider appropriate acceptance criteria for the analysis of mRNA by hybridization methods (bDNA and RTqPCR)?

\section{Hybridization HPLC-FL \& LCMS/HRMS assay of oligonucleotides \& comparison with hybridization ELISA}

What principles apply for hybridization HPLC-FL and LCMS/HRMS assays versus hybridization ELISA for oligonucleotides bioanalysis? What are the advantages and limitations of LCMS/HRMS analysis for quantitation of oligonucleotides? Is anything new from the recommendations in the 2017 White Paper Part 2 [17]? Extraction technology for LCMS involving immunoprecipitation to enrich the target is critical (immuno-MS). Do we understand selectivity of capture probes in hybridization? In the absence of SIL-IS, what are the best practices for internal standard response and reporting using LCMS for oligonucleotides? How can sensitivity of the current LCMS/HRMS setup be further increased? Is this really needed and what is expected to be needed in future? 


\section{Discussions, consensus \& conclusions}

Small molecule regulated bioanalysis \& peptides by LCMS

What ISR/ISS is telling the global bioanalytical community about its methods/procedures

Standards and QC samples used during validation may not mimic the actual study samples. Differences in protein binding, back-conversion of known and unknown metabolites, sample heterogeneity or concomitant medications may affect the accuracy and precision of the analyte quantitation in incurred samples during processing and storage. It is therefore recommended by both EMA and FDA $[19,20]$ to evaluate method reproducibility for analyte quantitation in incurred samples by reanalysis of study samples.

It was predicted that generic applications with bioequivalence studies carried out before the ISR requirement became common practice would not include ISR data. To address this, in the EMA questions and answers: positions on specific questions addressed to the Pharmacokinetics Working Party [24], considerations regarding a potential justification for the lack of ISR data have been described only in the case of data that pre-dates guidance. This includes possible metabolite back conversion, other ISR data obtained in the same laboratory with the same analytical method, an examination of data from repeat analysis, and the pharmacokinetic data, including the $90 \%$ CIs, obtained in the submitted bioequivalence study.

Over the decade in which ISR has been an integrated working practice within the bioanalytical community, the failure rate has appeared to be low across the industry. The low failure rate demonstrates that most methods, with few exceptions, are rugged and reliable. This raises questions whether ISR has helped at all in improving assay performance and where it is most valuable/critical to conduct routinely. If designed correctly, it can act as a quality check of all runs as well as the processes in use around sample analysis (sample mixing, aliquot issues, analyst to analyst variability). Attendees agreed that most issues are procedural and not scientific (i.e., not due to the methods).

Extensive discussions have taken place over the years at WRIB and within the GCC closed forums, where the global bioanalytical community provided recommendations in an effort to clarify how failed ISR evaluations should be handled [25,26].

Several discussions took place regarding ISR investigations for individual sample outliers or 'flyers' - that is, ISR values that differ by $>50 \%$ from the original values. Over the last several years, it has been common place for companies to investigate the reason for these individual outliers, following procedures described in SOPs. In some cases, the outcome of the investigation may result in the reporting of the investigation repeat value instead of the original value. In such cases, reporting of a repeat value has been based on the outcome of the investigation process, whereby the original value was shown to be erroneous. There has, however, been an increasing number of regulatory deficiency letters received, in cases when an original value was replaced. The consensus reached was that while an investigation may be merited, the original value should still be the final reported value. Additionally, it was also agreed that rather than focus on individual ISR outliers, investigations should be conducted in cases of overall ISR failures associated with a specific batch/batches, specific subject(s) and instruments. For example, if ISR meets the acceptance criteria yet shows large or systematic differences between results for multiple samples (e.g., all samples from one subject fail, all samples from one run fail) this may indicate analytical issues and it is advisable to investigate this further.

It is clear that an investigation is required to identify the cause of the failure, as well as a priori criteria on what to report after the investigations. In the case of failing ISR for a pivotal study (e.g., a BE study), a root cause may provide insight into the data as a whole to determine if the study could be salvaged. An open dialogue with regulators is encouraged and transparency in reporting is a must.

An extension of the ISR discussion has been ISS. The 2012 and 2015 White Papers in Bioanalysis include recommendations on ISS. These recommendations were confirmed and considered still valid $[5,10]$.

An attempt to harmonize the number of samples to reassay as part of ISR was also discussed. The approach outlined in the EMA and Japan MHLW guidelines [20,21] is based on using 10\% of the first 1000 samples and 5\% of the remaining samples. The FDA draft guidance [19] states that ISR should be performed on 7\% of incurred samples regardless of study size. The consensus was to wait for the approach described in the upcoming ICH M10 BMV guidance [27].

Post-discussion note: i0t should be noted that since the 2018 WRIB discussions, the final revised FDA BMV guidance has been released [28], harmonizing the ISR requirement with the EMA and MHLW guidelines on this issue, recommending using $10 \%$ of the first 1000 samples and $5 \%$ of the remaining number of samples. 


\section{Revisiting MIST (safety testing of drug metabolites)}

The FDA guidance regarding safety testing of metabolites was first released in 2008 and subsequently revised in 2016 [22]. Corresponding ICH guidances were issued in 2010 and 2013 [29,30]. These guidances have reinforced the importance of drug metabolite exposure for safety evaluations of NCEs, and their implementation has generated significant discussion within the industry, including potential strategies and challenges for quantitative metabolite bioanalysis [2,10,31]. The 2018 discussion focused on revisiting MIST following a decade of implementation.

There was consensus that the MIST guidelines are now mature and still appropriate and relevant for determining drug safety, with their requirements being addressed routinely in drug development plans. Application of MS techniques has continued to improve allowing for increasingly sophisticated profiling of metabolites in non-clinical species and early clinical studies in humans [32]. Although the MIST guidance specifically address non-clinical safety testing, understanding drug metabolism is also important for multiple aspects of drug development, including understanding pharmacology, PD responses and the potential for drug-drug interactions. Consequently, MIST strategies often overlap with analyses needed to define PK/PD relationships. It was noted that although MIST is small molecule centric, these principles could be applied to other types of molecule, particularly with the emergence of increasingly complex large molecule drug entities. However, many of these new entities are in the oncology area, and the MIST guidance does not generally apply to the development of drugs for patients with advanced cancer [22,33].

Although many small molecule drugs generate multiple metabolites, it is neither practical nor required to implement fully validated methods for all possible metabolites during early development phases. Strategies combining metabolite profiling and quantitative bioanalysis can be used to focus bioanalytical efforts on metabolites that are the most relevant and quantitatively important. Qualified but not fully validated methods for bioanalysis of metabolites can also be useful when metabolite exposure is considered borderline and to evaluate the need for further metabolite testing later in drug development [31].

\section{Therapeutic peptides - robust LCMS/HRMS \& hybrid LBA/LCMS methods}

Significant technological advances in MS have led to a shift in the bioanalytical support of therapeutic peptides from historically being quantified using LBAs to LCMS based analysis (e.g., triple quadrupoles, HRMS and hybrid LBA/LCMS).

High resolution instruments have been primarily utilized for qualitative assessments. Recent instrumentation advances have brought significant improvements in sensitivity and dynamic range of HRMS enabling quantitative analyses necessary for PK studies. The use of HRMS offers several advantages for bioanalysis and poses some challenges as well. Evolving recommendations on HRMS have been issued since 2015 [10,13,16]. However, there are still no current industry standards beyond the need for a validated method on a validated system if the results are to be used for regulated PK studies. The decision to use high resolution over unit resolution MS is analyte and matrix dependent. It was agreed that it is not common practice to sum multiple transitions to improve assay sensitivity for analysis. This approach has not been commonly attempted, but improvements in assay sensitivity and performance are possible as long as the approach leads to an increase in signal to noise ratio.

LCMS offers numerous benefits for the development of robust bioanalytical methods for detection of therapeutic peptides due to its high specificity, selectivity, large dynamic range and speed as LCMS methods are not dependent on generation of analyte-specific reagents that are required for LBA and LBA/LCMS hybrid methods. However, therapeutic peptide quantitation using LCMS can still be challenging when poor sensitivity and selectivity, peptide instability, non-specific binding and low recovery are observed. Some of these challenges can be addressed by utilizing IA purification during sample preparation for analyte enrichment. Over the past few years, the hybrid LBA/LCMS platform has become the method of choice for peptide bioanalysis when extra sensitivity (i.e., low $\mathrm{pg} / \mathrm{ml}$ LLOQs) and selectivity are needed. However, selection of the best IA capturing antibody and IA platform can be very challenging due to difficulties in obtaining capture reagents from consistent and reliable sources.

\section{Sophisticated bioanalytical methods that drive the quality of PKPD \& PBPK modeling}

The pharmaceutical industry has seen a steady increase in importance of various M\&S approaches to guide the decision-making process in drug discovery and development. The range of M\&S approaches employed ranges from simple PKPD models in preclinical species to complex Quantitative Systems Pharmacology and pharmacometric models. An important aspect in the use of M\&S is the fact that the quality of the predictions and their output data 
can only be as good as the quality of the input data and therefore the quality of the assay that is used to generate these data is critical. It is consequently very important to develop sophisticated and rugged bioanalytical methods to determine appropriate input parameters.

Modeling and simulation exercises mostly start with a biologist and a modeler to the exclusion of a bioanalytical scientist, an approach which tends to overlook the importance of the assay quality and its limitations. However, in order to build a useful model, the bioanalytical data quality must be adequate to answer the biological question. Bioanalysts can provide input on different assay options such as sensitivity and dynamic range, ensuring that the biological noise can be differentiated. In working as a team, method performance requirements can be determined to avoid the introduction of unnecessary variability that could impact the model. If the pharmacological variability is anticipated to be high, the assay performance criteria can also be more flexible. It might not be necessary to develop a fully validated method and the rigor of assay qualification/validation strongly depends on how the data will be used during the drug development process. For example, exploratory biomarkers for hypothesis building require assays with a different degree of characterization than bioanalytical assays which are being used to assess efficacy or safety endpoints during clinical development. Bioanalysts should contribute to what is measurable from a bioanalytical standpoint (e.g., feasibility) as well as the rigor that is needed to establish the method.

Next wave of automation \& next wave of issues to overcome: impact on regulated bioanalysis \& pros/cons of emerging automation in a regulated environment

Pressures on development costs have continued to drive innovation and disruptive business models in the pharmaceutical industry. Automation is one of the solutions that has been routinely sought after to relieve some of these pressures. Historically, automation has freed the bioanalyst from the routine aspects of analysis. However, the next wave of bioanalytical automation presents a significant degree of process improvement enabling enhancements across the range of analytics from sample preparation through instrumental analysis to point of care solutions. Panelists indicated that semi-automation is consistently in place in bioanalytical laboratories, but the level of automation varies, largely due to the high cost of implementation.

With the next level of innovation comes a host of issues that will need to be addressed as these automated solutions become embedded in the regulated bioanalytical environment. Some cons discussed for various technologies (e.g., liquid handling systems, automated immunoassays) include the need for manual interventions, the cost of disposables, the need for specialized training, increased maintenance and validation, and, as mentioned above, cost of implementation. Additionally, there can be challenges with sample reconciliation. Finally, some scientists are still hesitant to relinquish full control over precious samples. Benefits of automation include reduction of human error, increased throughput, reduced reagent use, increased flexibility and reduced variability. Another consideration is that automation changes how studies are inspected by regulators. The software and equipment play an important role in the conduct of the study so will require suitable validation, which would be reviewed on inspection.

The move towards automation is often viewed as dependent on the number of samples that need to be assayed. However, for quantitative bioanalysis where lots of studies are anticipated, such as in the high throughput screening arena, the return on investment can be worth the initial implementation costs resulting in lower fixed long-term costs. To realize the full potential of the next wave of automated analytical solutions, a step-wise approach incorporating proven individual task-based automation is required leading to modularized formats which can be combined to result in end-to-end bioanalytical workflows. Once established, these end-to-end bioanalytical workflows can be adopted and industrialized allowing for improved capacity with reduced resources, freeing scientists to invest in new techniques and skills more readily. In addition, end-to-end or other partially automated bioanalytical workflows should be fully developed and easy to externalize, allowing for increased CRO uptake while minimizing the burden of redevelopment once externalized.

By use of a continual process improvement cycle where analytical bottlenecks are identified and automated solutions can be provided, evaluated and embedded after being deemed beneficial, we can, as analytical scientists, continue to improve the science and quality of the bioanalytical support we provide.

\section{Small biomarkers by LCMS \\ Biomarker assay validation for small molecule biomarkers by LCMS: alignment between the recommendations of the C-Path White Paper o the 2016/2017 White Papers in Bioanalysis (Part 1)}

Currently there is much discussion around the challenges of targeted quantitation of biomarkers to support drug development. Many of these challenges are associated with protein biomarkers. Among them, the heterogeneity 
of the endogenous protein, with PTMs and varied possible isoforms, complicate the analysis and interpretation of results. For small molecule biomarkers, we can obtain well-characterized and representative reference materials because there are fewer structural modifications for small molecules compared to proteins. Chromatographic approaches dominate such applications, and linear response functions for methods employed are prevalent. Still, the analytical challenge of endogenous analytes of interest persists with small molecule biomarkers, but this can be addressed in a consistent manner using a surrogate matrix or a surrogate analyte approach [34]. All biomarker bioanalysis should be differentiated from xenobiotic drug bioanalysis, but small molecule biomarkers represent a class that is more amenable to established bioanalytical practice than protein biomarkers. While the basic principles are similar, not all aspects of BMV guidance may always be applicable and should be considered on a case-by-case basis. Therefore, small molecule biomarkers should be considered separately from protein biomarkers.

Thus, development of prescribed guidance for bioanalysis of biomarkers presents a great challenge, and the pace has been understandably slow. In the interim, several articles and White Papers have been published to attempt to fill the gap [12,13,15-18,35-37]. In 2017, the Biomarker Assay Collaborative Evidentiary Considerations Writing Group, which includes contributing authors from US FDA, published what is known as the C-Path White Paper [23]. The C-Path White Paper was originally focused on BAV for LBA Assays. However, small molecule biomarkers are very important as surrogates for disease diagnosis, progression, and prognosis and it was agreed that the foundation of biomarker qualification/validation by LCMS is in scope of the White Paper. As a consequence, the paper was expanded to cover the similarities and differences between LBA and LCMS methods.

US FDA will use the C-Path White Paper as part of a scientific consensus framework for two guidance documents, safety biomarker qualification and biomarker method validation. However, until these guidance documents are available, a revised version of the White Paper has been issued in 2018 [66] which includes several discussion points described below.

Although referenced in the C-Path White Paper, matrix effect/factor and ion suppression/enhancement requirements are already captured in the BMV guidance. Criteria to define the LLOQ and QC levels in the calibration curve are already defined and additional details will be included in an appendix. Similar criteria for total allowable error for biomarkers assayed by LBA and LCMS are also recommended as the approach is based on the clinical utility rather than the specific analytic technology. When using a surrogate matrix approach, it is good practice to perform parallelism experiments using samples containing the endogenous analytes in method validation, but it is not required. Dilution linearity tests can be a valuable probe of endogenous analyte selectivity in the biological matrix, and as such may be a useful method development tool for assay characterization. When applied, it is best to use matrix from multiple individuals to test the method. For BAV using an LCMS assay, accuracy and precision using three separate lots of matrix is recommended. Although it has been suggested that longitudinal QCs are favored over ISR for small molecule biomarkers, longitudinal QCs may not be representative of patient populations, and should not completely replace ISR. Finally, stability evaluations of the biomarker in presence of the drug (i.e., co-admin stability) are not necessarily required. Ultimately, the science must drive biomarker assay validation strategy in a way that is considerate of the underlying biology, analytical methodology, and the appropriate quality of the generated data.

\section{Boosting small molecule biomarker assay sensitivity}

Lack of authentic matrix samples with undetectable or low levels of intended endogenous analyte can present a challenge when developing biomarker assays within the linear response range of instruments. A feasible assay LLOQ can be determined to be $>20 \%$ of the endogenous level of the standard curve matrix when using standard addition method. Using a cleaner lot as standard curve matrix is recommended.

Some indications require very sensitive assays with LLOQs - for example, $\sim 10 \mathrm{x}$ lower than endogenous levels to be informative for PD response. A case study involving the sphingolipid glucosylceramide (GL-1), used as a surrogate biomarker for enzyme replacement and substrate reduction therapies in patients with Niemann-Pick, Gaucher and Fabry diseases, was presented in order to demonstrate this challenge [38]. Due to high endogenous levels of this sphingolipid, it was difficult to achieve the desired sensitivity for the intended clinical application using an authentic matrix approach. Therefore, a surrogate matrix approach with delipidized plasma was utilized. Evaluation of parallelism and relative matrix effect were performed in assay qualification/validation. When a surrogate matrix is used for standard curve preparation, relative matrix effect can be evaluated at three levels: (a) spiked analyte in the surrogate matrix alone; (b) the endogenous matrix only (at least six lots if endogenous level is higher than LLOQ); and (c) spiked analyte to authentic matrix (the same six lots) to evaluate passing accuracy at 
each level and to demonstrate that spiked analyte can be correctly added to endogenous analyte. IS consistency, its extraction recovery and matrix effect that impact the assay performance should be examined along with the analyte in surrogate matrix and in authentic matrix. The IS consistency criteria can be set per assay specifications to meet the purpose.

\section{Recent developments in the quantification of individual bile acids as biomarkers for drug induced liver injury}

To assess the potential of IBA and their profiles as mechanistic biomarkers of liver injury for humans in real world situations, a case study was presented to stimulate the discussion. This study interrogated samples collected under minimum controlled conditions (i.e., fasting). LCMS methodology was established to analyze nine IBA, generate reference ranges, and examine effects of age, gender, and ethnicity for each IBA. Furthermore, the ability of IBA and their profiles to detect hepatic injury in subjects with a broad range of liver impairments was evaluated [39]. To date, the study utilized the largest cohorts to evaluate IBA as biomarkers. Results showed that the serum concentrations of IBA, such as GCA, GCDCA, CDCA, and TCDCA were significantly increased. This report is the first to describe ethnic differences in serum concentrations of IBA. The conjugated bile acids displayed greater differences between healthy subjects and subjects with hepatic impairments than non-conjugated bile acids. Furthermore, the subjects with hepatic impairments exhibited distinct profiles (signatures) of IBA that clustered subjects according to the nature of their liver impairments. Although additional studies are needed, the data suggested that the analysis of IBA has the potential to become useful for detection, and potentially differentiation, of various forms of liver injury.

The consensus was that the best/current method development strategies to overcome the high variability of biomarker bile acids and optimize method selectivity and sensitivity were to use wide dynamic ranges and group the analytes based on functional similarity and analytical performance. HRMS could then be used to check selectivity/specificity and improve sensitivity for unconjugated bile acids.

As discussed in the above section, the surrogate matrix approach for IBA analysis is a viable alternative to measure human plasma samples as long as parallelism experiments are successful.

\section{Endogenous small molecule biomarkers for renal \& hepatic transporters}

Transporters play a fundamental role in DMPK/ADME and are presently studied using probe drugs [40]. In vitrobased transporter DDI evaluations result in a high number of false positive rates $(\sim 30 \%)$ and categorize new drugs as potential candidates for dedicated clinical DDI evaluations. Rapidly advancing drug transporter biomarker sciences suggest that selected endogenous compounds, detectable in human plasma and urine, may serve as biomarkers for specific transporters. Among them, coproporphyrin isomers, coproporphyrin-I (CP-I) and coproporphyrin-III (CP-III), bile acids, and N1-methylnicotinamide (NMN) have been evaluated as possible candidate biomarkers for organic anion-transporting polypeptides (OATP1B1 and OATP1B3), organic cation transporters (OCT1 and OCT2), and multidrug and toxin extrusion proteins (MATEs) [41-44]. More studies using these biomarkers are needed to facilitate collaboration within industry and evaluate clinical utility of these in-vitro DDI predictions.

If parallel studies demonstrate that endogenous biomarkers give the same DDI risk projections as dosing probe drugs, then reservations to reduce or completely eliminate the studies which involve dosing of the probe drugs would be overcome. It may be possible to use existing PK samples for endogenous biomarker profiling or DDI projections, however, there must be permission via the informed consent. Furthermore, biological effects such as timing of collection, food, diurnal changes must be considered. The preferred approach for early stage exploratory biomarker work is to use multiplexed HRMS assays for profiling multiple endogenous biomarkers [45]. The development of a highly selective and sensitive UHPLC-HRMS assay affords options for earlier characterization and clinical safety projections for OATP1B1/3 [45-49], selected bile acids [50] and NMN-mediated DDIs [51] along with pharmacokinetic analyses of drugs as part of FIH clinical studies. In MS-based methods, it is preferred to use SIL-IS for each endogenous biomarker; however, structural analogues can be used as an alternative as long as they are properly validated.

\section{Oligonucleotides \\ Recent advances, future trends \& impact on oligonucleotide bioanalysis}

Analysis of oligonucleotides from biological samples is a challenging analytical task. Therapeutic oligonucleotides are either single or double stranded DNA- or RNA-molecules with a specific nucleotide sequence. Chemical modifications reduce endo- and/or exo-nuclease mediated degradation thereby improving in vivo drug metabolic 
stability. They differ in length from approximately 15 up to $>1000$ nucleotides, are sometimes conjugated with different ligands and may be formulated in LNPs or other delivery systems, such as viral vectors. Due to the heterogeneous nature of the analytes and their exposure in matrices that can vary significantly in nature, no single bioanalytical approach can be applied to cover all the different therapeutically relevant oligonucleotide classes (e.g., ASO, siRNA, miRNA mimics or inhibitors, aptamers, decoys, sgRNA, mRNA, immuno-stimulatory oligonucleotides) [52-56].

Gene therapy attempts to modify a genetic sequence in order to help fight a disease. This may be done by replacing or inactivating a mutated gene that causes a disease or by introducing a new gene into the body. Despite the fact that several GTMPs have been approved and many are in clinical investigation, safety and sustainable efficiency are still main concerns to drug developers. The pharmacokinetic behaviors and the immunogenicity of both GTMPs and the expressed gene moieties are essential for understanding pharmacology and safety. Several regulatory documents exist to provide guidance on gene therapy studies [57-59].

To enable accurate and efficient PK/PD evaluations and immunogenicity assessments of oligonucleotides, a thoughtful bioanalytical strategy has to be established and implemented prior to supporting preclinical and clinical studies. With more technological advances, bioanalytical scientists can now select analytical tools from a number of options to develop/validate specific, accurate, reproducible and sensitive assays and generate meaningful data [20,28].

Multiple methods are required for adequate characterization of the PK of ASOs and siRNAs. LCMS, LBA (hybridization ELISA), RT-qPCR (or equivalent), FL-HPLC are all options; drivers of analytical platform selection are associated to the objective of the study and typically depends on the specificity, sensitivity and matrix. If multiple bioanalysis platforms are used, regulators do not expect identical data from each, but comparability or lack of comparability must be understood. Indeed, some assays only detect full length drug, while others may include full length drug and drug metabolites (due to exo-nuclease activities).

For both PK and toxicity studies, if a formulation using novel excipients (e.g., lipids, PEGs, or polymers) or a delivery vehicle (like viral delivery) is used to administer the oligonucleotide, it is advisable to understand the exposure of these formulation components. The data will help to fully understand the safety liability of the drug and its novel carrier and this is considered of greater importance for chronically dosed drugs.

If the oligonucleotide has a poor stability profile, such as in the case of an mRNA therapeutic which is highly unstable in the presence of nuclease enzymes, an encapsulating formulation such as a LNP may be used to protect the drug and prolong its exposure. During method validation and routine sample analysis, using the unformulated oligonucleotide may not be preferred due to stability concerns. Therefore, the LNP formulated drug product can be used for the preparation of matrix matched standard curves and QCs, provided that the standard is sufficiently characterized in terms of stability, concentration and impurities.

Finally, it was agreed that oligonucleotide drug projects should be reviewed against the principles of MIST [22] and characterization should be considered on a case by case basis as each oligonucleotide class will have different requirements. Compared to small molecules, the major routes of metabolism are substantially different and the metabolism of oligonucleotides can be more predictable. Data and further scientific and regulatory understanding are needed before such analysis can be reduced or eliminated for oligonucleotides.

\section{The bioanalytical challenge of oligonucleotide biodistribution: current industry approaches for biological fluids/tissues, unsolved issues, unanswered questions \& future perspectives}

The bioanalysis of oligonucleotide drugs such as ASOs is an essential component for a proper understanding of the PK properties of these molecules. A full characterization of the PK of ASOs must account for the several orders of magnitude difference in concentrations between high accumulating tissues, such as kidney cortex and liver with concentrations in the $\mu \mathrm{g} / \mathrm{g}$ range, and the trough plasma with concentrations in the $\mathrm{ng} / \mathrm{ml}$ range or lower, which often necessitates the use of multiple assays such as LBAs and LCMS methods. The different levels of sensitivity required for different assays means that each method must address the specific needs of each matrix.

Administration of modified mRNA enables expression of virtually any desired endogenous or exogenous protein. Some challenges include low tissue targeting (RNA permeability to penetrate cell membrane of target tissues), low expression, and short half-life of the product [60,61]. The clinical success of mRNA drug platforms requires efficient delivery to the desired tissues.

Viral vectors and LNPs are frequently used drug delivery technologies. Understanding the complex biodistribution and pharmacological activity profile of LNP delivered modified mRNA and associated expressed protein is essential to fully comprehend the impact on both efficacy and safety of the modality. As such, the bioanalyti- 
cal requirements for a single modified mRNA study may include the measurement of mRNA, protein or other drug-related material in numerous tissues and matrices.

Quantitative analysis is mainly conducted by LCMS, hybridization-ELISA (based on S1 nuclease protection), RT-qPCR (or equivalent), bDNA or AEX-HPLC with fluorescence detection (DNA- or PNA- probe HPLCAssay). Depending on the analytical technique and the biological matrix, the stabilization and extraction of the oligonucleotide could be the most critical step, one that is very complex and time consuming, in order to deliver highly accurate and sensitive data. In some instances, multiple methods would be required for adequate PK characterization (e.g., ASOs). Sensitivity needs and matrices requirements are the other drivers of analytical platform selection.

Methodologies for measuring tissue concentrations can be validated or qualified depending upon the intended use of the data generated. Validation would be required in situations where critical clinical dosing decisions are made based upon the tissue data, but otherwise qualifications could be sufficient. When a method is qualified, the minimum critical characteristics to evaluate for quantitative nucleotide drug measurement in tissue homogenates include at least one batch for assay accuracy and precision, sensitivity, matrix selectivity and, depending on the oligonucleotide class and technology choice, it may be relevant to evaluate metabolite interference. Tissue recovery should be evaluated during method development when appropriate, such as when extensive or detailed extraction procedures are used. Care should be taken to manage the potential for blood contamination and thorough washing of the tissue before freezing is advised. For some tissues, it may not be possible to acquire sufficient blank matrix, therefore a surrogate matrix (typically a more abundant tissue) can be evaluated and used instead with justification.

The best way of correlating serum/plasma analyte concentrations with tissue levels is to use animal data to determine the ratio of serum/plasma to tissue, followed by allometric scaling of the serum/plasma levels then using the serum/plasma to tissue ratio to estimate tissue levels that cannot be obtained in humans. Confirmation of the adequacy of the scaling should be done whenever human tissue is accessible. Understanding the difference in drug targeting between animal and human will also help estimate human tissue distribution with biopsy samples typically not available or with very limited quantities.

\section{Challenges with determination of oligonucleotide immunogenicity}

Biological drugs often elicit immune responses and result in the production of ADA. Drug-bound ADA can lead to loss of efficacy, immune toxicity and adverse events. ADA can also alter the PK properties of the drug through clearance or neutralization of the pharmacology of the drug. To keep track of their impact on PK and PD parameters as well as safety, ADA are monitored in clinical trials using appropriate immunoassays.

Risk-based strategies and general guidelines for immunogenicity assessments based on the structural complexity, immunogenic potential and other practical considerations, including the availability of appropriate controls, PK profiles and API half-life should be considered. Challenges include red blood cell partitioning, serum protein binding and macrophage sequestration that can potentially impact PK.

Given the intracellular site of action of ASOs, properly defining ADA at the outset of development can lead to structuring the assays differently. Not all ADA+ responses are neutralizing. However, an ADA bound to drug may change the biodistribution, PK and PD. If the presence of ADA reduces the PD (being analyzed by a high-quality quantification method), then the ADA can be considered neutralizing, but conversely an ADA which positively affects PK, and potentially PD, could be considered enhancing. It is therefore important to look at the PK/PD impact during the assessment. Development of a ligand binding assay to determine whether ADA have neutralizing activity may not be suitable given the intracellular drug mechanism of action.

Where a potential pre-existing immune system liability is suspected, such as an ADA response to PEG (a component used within an LNP formulation), then pre- and post-treatment assessments are advised regardless of whether cytokines or antibodies are being measured. When complex delivery systems are used to deliver oligonucleotides, it may be necessary to deploy a number of different ADA assays to fully assess each component that has the potential to invoke an immunogenic response. For the development of ADA assays, the same principles apply as described in guidance for ADA to protein products. The establishment of proper cut-points for both LNPs, gene therapy via viral vectors, and ASOs/siRNAs is extremely important. The difficulty of developing positive controls for antibody assays, especially ASOs and siRNAs, should not be underestimated.

With mRNA/gene therapy that results in expression of a protein, assays that assess the development of ADA to the encoded protein are needed. Even with a short duration of exposure, development of an anti-siRNA or ASO ADA assay may still be required because multiple doses may cause the development of ADA. Also in a single dose 
study, understanding immunogenicity remains important especially if infusion and/or injection related reactions which may or may not be ADA mediated are present. Potential NAb may also cross-react with endogenous low abundant proteins.

It is unclear at this time whether there is a consensus regarding the sensitivity and drug tolerance requirements of immunogenicity assays for oligonucleotides. However, sensitivity of an ADA assay is mostly dependent on the positive control antibody. Ideally, sensitivity should be determined against a range of different antibodies with different binding affinities to the target oligonucleotide. The best effort should be made to produce a good quality assay that gives the highest possible chance of detecting a true ADA response against the biotherapeutic. The drug tolerance requirements of the assay need to be determined for each therapeutic based on the intended dosing regimen and subsequent exposure at the ADA sampling time points. Generation of surrogate animal positive ADA control may be challenging to demonstrate the expected sensitivity.

\section{Branched DNA \& RT-qPCR/stem-loop qPCR assays: an in depth understanding of method development \& validation challenges in the quantification of oligonucleotides}

Small oligonucleotides ( $\mathrm{n} \approx 20$ bases) could be relatively easily quantified by well-established LCMS or ligand binding type methods; however, mRNA-based therapies utilize substantially larger polynucleotides (up to several thousand bases long), making their quantification by LCMS a challenging task. It becomes especially difficult when assessing the distribution of the mRNA drug product into tissues, as the effects of the matrix can impact assay performance (e.g., differing in tissue recovery or nuclease enzyme content affecting stability).

RT-qPCR (or equivalent) and bDNA are the most commonly used methods for RNA quantification. While being widely used, PCR methodologies are often not readily available in traditional regulated bioanalysis laboratories/CROs. Consideration should be given to bringing this technology into laboratories if they are to be used routinely for quantitative oligonucleotide bioanalysis. Implementation should include rigorous analyst training. There was concern that the qPCR-based methods could be impacted by matrix effects which may limit its usefulness to assess tissue biodistribution. Moreover, as primer selection usually varies from sequence to sequence, it is hard to ensure that the readout reflects the concentration of the entire mRNA sequence. For small oligonucleotide drugs, a stem-loop primer is used due to limited length of target oligonucleotide for primer binding.

With that, bDNA appears to be a favored method for mRNA quantification, although RT-qPCR (or equivalent) is also a reasonable option. bDNA has been successfully used to assess mRNA 'drugs' and downstream events in blood and tissues [62,63]. As with TaqMan RT-qPCR approaches, the application of a multiplex version of the bDNA assay allows the assessment for multiple mRNAs simultaneously.

Appropriate acceptance criteria for the analysis of mRNA by hybridization methods (bDNA and qPCR/RTqPCR) have not been put forth by regulators because there is not yet enough validation data available to inform guidance. However, it is recommended that bioanalysts strive to meet LBA guidelines where feasible, and generate evidence to take back to regulators as a community.

\section{Hybridization HPLC-FL \& LCMS/HRMS assay of oligonucleotides \& comparison with hybridization ELISA}

Therapeutic oligonucleotides, such as ASOs, often differ from natural oligonucleotides through modifications in the backbone. The phosphorothioate linkage in the backbone was introduced to enhance nuclease resistance and improve cellular uptake. Additional modifications are introduced to enhance target delivery and potency. The reduced effective dosages inevitably generate an increased demand for sensitive, accurate, efficient, and reliable assays for oligonucleotide characterization and quantitation. Traditionally, the gold standard bioanalytical methods - hybridization ELISA/PCR technology - provided very sensitive assays. However, orthogonal LCMS technology complements existing assays, allowing for fast method delivery in early development.

The advantages of using LCMS/HRMS analysis, recommended in the 2017 White Paper [17], include better specificity, improved separation of any metabolites from the full-length molecule, and reasonable sensitivity. However, throughput can be lower than traditional LCMS. Like other techniques, an appropriate IS is needed but stable labeled oligonucleotides have not been routinely used due to the perceived difficulty for production [64]. Typically, a surrogate oligonucleotide can be used as an IS by attempting to match chemistry and stereochemistry to obtain the same extraction efficiency as the analyte. In an effort to increase sensitivity, serial liquid-liquid or SPE extractions may be an option for both plasma and tissue samples. Alternatively, immunoaffinity enrichment of targets may also be a viable method to decrease background interferences, but this approach may have additional 
challenges. Hybridization methods require specific and high-affinity probes as the specific binding to the target is critical. Shorter (n-1, n-2) oligonucleotides often bind to the probes, leading to erroneous results/data.

HPLC-FL has good tolerance to structural modifications and an IS is not typically required. Although this method offers promising characteristics including high sensitivity, large dynamic range, simple sample preparation and may provide similar selectivity to LCMS in some circumstances, limited data is available to support these claims. However, the long run times (10-30 min/sample) and chromatographic separation of $\mathrm{n}-1$ and $\mathrm{n}-2$ oligonucleotides seems challenging.

These methods are expected to meet regulatory validation criteria. For binding-based assays including hybridization ELISA, HPLC-FL, and qPCR/RT-qPCR, LBA assay criteria are suitable since these methods require binding steps for probe or primer binding to the target nucleotide. For binding-free LCMS assays, chromatographic assay criteria are considered appropriate. As more data are generated in late stage drug development, regulators and industry may be able to better define assay performance expectations in the future.

\section{Recommendations}

Below is a summary of the recommendations made during the $12^{\text {th }}$ WRIB.

\section{Small molecule regulated bioanalysis \& peptides by LCMS}

\section{ISR/ISS}

- An investigation is required to identify the cause of an ISR failure, as well as a priori criteria on what to report after the investigations;

- An open dialogue with regulators is encouraged following ISR failure of a pivotal study;

- Transparency in reporting ISR failures is a must;

- ISR investigations for individual sample outliers may be merited, however the original value should still be the final reported value. Investigations should also be conducted in cases of overall ISR failures, ISR failures associated with a specific batch/batches, specific subject(s) and instruments;

- The use of ISS should be issue driven as per previous recommendations [5-10].

2. There was consensus that the MIST guidelines are still appropriate and relevant for determining drug safety. Advances in LCMS techniques allow for increasingly sophisticated metabolite profiling in the early stages of drug development, hence enabling bioanalytical methods to be focused on the most relevant metabolites. In future, MIST principles may also need to be applied to novel and increasingly complex molecular entities now being investigated as potential drugs;

3. The use of quantitative HRMS over unit resolution MS is analyte and matrix dependent. One option to improve sensitivity for therapeutic peptides analysis can be to sum multiple MRM as long as specificity/selectivity has been carefully evaluated; the signal to noise ratio needs to increase when summing multiple transitions;

4. It is very important to develop sophisticated bioanalytical methods to determine critical input parameters for PKPD models. Bioanalysts can contribute to what is measurable from a bioanalytical standpoint (e.g., feasibility) as well as the rigor needed to establish the method;

5. Semi-automation is consistently in place in bioanalytical laboratories, but the level of automation varies, largely due to the cost of implementation, the need for specialized staff and the amount of samples that require assaying at any one time.

\section{Small molecule biomarkers by LCMS}

6. Matrix effect/factor and ion suppression/enhancement requirements are already well-captured in the BMV guidance. No additional requirements for small molecule biomarkers are expected;

7. Similar criteria for total allowable error for biomarkers assayed by LBA and LCMS are recommended;

8. When using a surrogate matrix approach, it is good practice to perform parallelism experiments in method validation, but it is not required. Dilution linearity should be tested during method development for assay characterization; it is best to use matrix from multiple individuals to test the method;

9. For BAV using an LCMS assay, accuracy and precision using three batches is recommended;

10. The value of ISR cannot be fully replaced by use of longitudinal QCs for small molecule biomarkers;

11. While the stability assessment of the biomarker in presence of the drug (i.e., co-admin stability) is generally not required, the potential for drug-biomarker interaction should be carefully evaluated on the basis of biology/chemistry context; 
12. Evaluation of parallelism, matrix effect and IS consistency is critical in determining whether special extraction conditions may be required to achieve equivalent recovery from both authentic and surrogate matrix;

13. A feasible assay LLOQ should be at least $>20 \%$ of endogenous levels when using the authentic matrix to prepare standard curve. Using a cleaner lot as standard curve matrix is recommended;

14. If surrogate matrix is used for the standard curve, relative matrix effect from at least six different lots should be evaluated for biomarker assay qualification/validation;

15. IS consistency, its extraction recovery and matrix effect should be examined along with the analyte in surrogate matrix and authentic matrix if the surrogate matrix approach is used to determine their impact on the assay performance;

16. The best/current method development strategies to overcome the high variability of biomarker bile acids and optimize method selectivity and sensitivity are to use wide dynamic ranges and group the analytes based on functional similarity and analytical performance. HRMS can be used to check selectivity/specificity, and improve sensitivity for unconjugated bile acids;

17. The use of matrix from a different species is a viable alternative to measure human plasma samples for IBA analysis as long as parallelism experiments are successful;

18. If parallel studies are conducted to demonstrate that endogenous biomarkers give the same DDI risk projections as dosing probe drugs, then there would be no reservations to reduce or remove the studies which involve dosing of the probe drugs:

- Existing PK samples for endogenous biomarker profiling or DDI projections can be used provided there is permission via the informed consent.

19. In MS-based methods, it is preferred to use SIL-IS for each endogenous biomarker; however, structural analogues can be used as an alternative as long as assay performance is acceptable.

\section{Oligonucleotides}

20. To enable accurate and efficient PK/PD and safety evaluations and immunogenicity assessments of oligonucleotides, pre-establish and implement a thoughtful bioanalytical strategy to support preclinical and clinical studies;

- Technology selection should be based on the objectives of the study and the data needed to support these objectives. LCMS, hybridization-ELISA assay, qPCR (or equivalent), bDNA or DNA- or PNA probe-HPLC-FL assays are all options depending on class of oligonucleotide, sensitivity needs and matrix requirements. If multiple platforms are used, regulators do not expect identical data from each, however comparability or lack of comparability must be evaluated and understood;

- For both PK and toxicity studies, if a formulation using novel excipients (e.g., lipids, PEGs, polymers, etc.) or a delivery vehicle (e.g., viral delivery) is used to administer the oligonucleotide it is advisable to understand the exposure of these formulation components. In addition, the immunogenicity of the delivery system, carriers, or excipients may need to be established;

- The best way of correlating serum/plasma levels of PK analytes with tissue levels is to use animal tissue data to determine the ratio of serum/plasma to tissues, followed by allometric scaling of the serum/plasma levels then using the serum/plasma to tissue ratio to estimate tissue levels that cannot be obtained in humans. Confirmation of the adequacy of the scaling should be done whenever human tissue is accessible;

- Whenever possible correlate immunogenicity and PK data;

- It can be beneficial to use formulated drug product for the preparation of mRNA standards and QCs to take advantage of the protection/stabilization properties provided by LNP formulations;

- The principles of MIST [22] should be assessed for oligonucleotides, when metabolism/catabolism may lead to potential efficacy and/or toxicity issues;

- Develop sensitive assays to monitor ADA. Not all ADA are neutralizing. If presence of ADA reduces PD and efficacy, then the ADA can be considered neutralizing. It is therefore important to look at the PK/PD impact during the assessment;

21. The bioanalytical requirements for a single modified mRNA (preclinical) study may include the measurement of the mRNA, protein or other drug-related material in numerous tissues and matrices;

- It is important to understand biodistribution as much as possible as well as the distribution of the protein that is expressed from the mRNA. Relevant tissues should be collected and studied (where feasible). 
22. When a tissue assay is qualified rather than validated, the critical minimum characteristics to evaluate in method qualification for quantitative nucleotide drug measurement in tissue homogenates may be technology and oligonucleotide specific and can include one batch for assay accuracy and precision, sensitivity, matrix selectivity and possibly metabolite interference. ISR and assessment of sample stability are not required for qualification and accuracy/precision does not need to meet BMV requirements:

- For some tissues, it may not be possible to acquire sufficient blank matrix, therefore surrogate matrix can be used instead with justification.

23. If there is a short duration of exposure, development of an anti-drug ADA assay is still required because multiple doses could cause the development of ADA:

- Pre- and post-treatment assays must be done the same way regardless of whether cytokines or antibodies are being measured. It is also important to properly establish cut-points; the same rules apply to both LNPs and ASOs.

24. Consideration should be given to bringing $\mathbf{q P C R}$ technology into laboratories if oligonucleotide assays will be needed. Implementation should include rigorous analyst training:

- It is recommended that bioanalysts strive to meet current LBA guidelines for the analysis of mRNA by hybridization methods (bDNA and RT-qPCR). These data can form the basis for future discussions with regulators regarding specific hybridization method criteria.

25. The same validation principles apply for hybridization HPLC-FL, LCMS/HRMS assays or hybridization ELISA for oligonucleotides bioanalysis. Each platform has its unique pros and cons:

- For LCMS/HRMS analysis, selection of an IS that matches chemistry and stereochemistry (if necessary) with the aim to obtain the same extraction efficiency as the analyte, is challenging;

- To increase sensitivity, double liquid-liquid extractions using phenol and chloroform combination or SPE, IA enrichment, or selecting multiple target ions are options but should be carefully evaluated;

- Hybridization methods require specific and high-affinity probes. Shorter truncated metabolites may bind to the probes leading to erroneous results/data with immunoassays;

- IS is not required for HPLC-FL methods, and they have good tolerance to structural modifications;

- Validation criteria for HPLC-FL, bDNA, and hybridization ELISA may follow LBA criteria since primer and DNA- or PNA-probe binding is needed. For binding-free LCMS assays, chromatographic assay criteria are more suitable. As more data becomes available from late stage drug development studies, regulators and industry should be able to better understand assay performance of these emerging technologies.

\section{Acknowledgements}

- The US FDA, Europe EMA, UK MHRA, Brazil ANVISA, Health Canada, Japan MHLW and Finland Fimea for supporting this workshop.

- C Beaver (Syneos Health), S (Julia) Cho (US FDA), J Kamerud (Pfizer), S Keller (Abbvie), F Garofolo (Angelini Pharma), J Marini (Janssen), A Musuku (Pharmascience), H Neubert (Pfizer), T Olah (Bristol-Myers Squibb), R Pillutla (Bristol-Myers Squibb), S Richards (Sanofi), L Stevenson (Biogen), A Torri (Regeneron), S Vinter (UK MHRA), J Welink (EU EMA), Y Xu (Alnylam) and E Yang (GlaxoSmthKline); for chairing the workshop and/or the white paper discussions.

- The Biogen Team (L Stevenson, C Stebbins, S Purushothama and D Mehta) for the pre-review of Part 3.

- All the workshop attendees and members of the Global Bioanalytical Community who have sent comments and suggestions to the workshop to complete this White Paper.

- Future Science Group as a trusted partner.

Financial \& competing interests disclosure

The authors have no relevant affiliations or financialinvolvement with any organization or entity with a financial interest in or financial conflict with the subject matter or materials discussed in the manuscript. This includes employment, consultancies, honoraria, stock ownership or options, expert testimony, grants or patents received or pending, or royalties.

No writing assistance was utilized in the production of this manuscript.

\section{References}

1 Savoie N, Booth BP, Bradley T et al. 2008 White Paper: the 2nd calibration and validation group workshop on recent issues in Good Laboratory Practice Bioanalysis. Bioanalysis 1(1), 19-30 (2009). 
2 Savoie N, Garofolo F, van Amsterdam P et al. 2009 White Paper on recent issues in regulated bioanalysis from the 3rd calibration and validation group workshop. Bioanalysis 2(1), 53-68 (2010).

3 Savoie N, Garofolo F, van Amsterdam P et al. 2010 White Paper on recent issues in regulated bioanalysis and global harmonization of bioanalytical guidance. Bioanalysis 2(12), 1945-1960 (2010).

4 Garofolo F, Rocci M, Dumont I et al. 2011 White Paper on recent issues in bioanalysis and regulatory findings from audits and inspections. Bioanalysis 3(18), 2081-2096 (2011).

5 DeSilva B, Garofolo F, Rocci M et al. 2012 White Paper on recent issues in bioanalysis and alignment of multiple guidelines. Bioanalysis 4(18), 2213-2226 (2012).

6 Stevenson L, Rocci M, Garofolo F et al. 2013 White Paper on recent issues in bioanalysis: 'hybrid' - the best of LBA \& LC/MS. Bioanalysis 5(23), 2903-2918 (2013).

7 Fluhler E, Hayes R, Garofolo F et al. 2014 White Paper on recent issues in bioanalysis: a full immersion in bioanalysis (Part 1 - small molecules by LCMS). Bioanalysis 6(22), 3039-3049 (2014).

8 Dufield D, Neubert H, Garofolo F et al. 2014 White Paper on recent issues in bioanalysis: a full immersion in bioanalysis (Part 2 hybrid LBA/LCMS, ELN \& regulatory agencies' input). Bioanalysis 6(23), 3237-3249 (2014).

9 Stevenson L, Amaravadi L, Myler H et al. 2014 White Paper on recent issues in bioanalysis: a full immersion in bioanalysis (Part 3 - LBA and immunogenicity). Bioanalysis 6(24), 3355-3368 (2014).

10 Welink J, Fluhler E, Hughes N et al. 2015 White Paper on recent issues in bioanalysis: focus on new technologies and biomarkers (Part 1 - small molecules by LCMS). Bioanalysis 7(22), 2913-2925 (2015).

11 Ackermann B, Neubert H, Hughes N et al. 2015 White Paper on recent issues in bioanalysis: focus on new technologies and biomarkers (Part 2 - hybrid LBA/LCMS and input from regulatory agencies). Bioanalysis 7(23), 3019-3034 (2015).

12 Amaravadi L, Song A, Myler H et al. 2015 White Paper on recent issues in bioanalysis: focus on new technologies and biomarkers (Part 3 - LBA, biomarkers and immunogenicity). Bioanalysis 7(24), 3107-3124 (2015).

13 Yang E, Welink J, Cape S et al. 2016 White Paper on recent issues in bioanalysis: focus on biomarker assay validation (BAV) (Part 1 small molecules, peptides and small molecule biomarkers by LCMS). Bioanalysis 8(22), 2363-2378 (2016).

14 Song A, Lee A, Garofolo F et al. 2016 White Paper on recent issues in bioanalysis: focus on biomarker assay validation (BAV): (Part 2 Hybrid LBA/LCMS and input from regulatory agencies). Bioanalysis 8(23), 2457-2474 (2016).

15 Richards S, Amaravadi L, Pillutla R et al. 2016 White Paper on recent issues in bioanalysis: focus on biomarker assay validation (BAV): (Part 3 - LBA, biomarkers and immunogenicity). Bioanalysis 8(23), 2475-2496 (2016).

16 Welink J, Yang E, Hughes N et al. 2017 White Paper on recent issues in bioanalysis: aren't BMV guidance/guidelines 'scientific'? (Part 1 - LCMS: small molecules, peptides and small molecule biomarkers). Bioanalysis 9(22), 1807-1825 (2017).

17 Neubert H, Song A, Lee A et al. 2017 White Paper on recent issues in bioanalysis: rise of hybrid LBA/LCMS immunogenicity assays (Part 2: hybrid LBA/LCMS biotherapeutics, biomarkers \& immunogenicity assays and regulatory agencies' inputs). Bioanalysis 9(23), 1895-1912 (2017).

18 Gupta S, Richards S, Amaravadi L et al. 2017 White Paper on recent issues in bioanalysis: a global perspective on immunogenicity guidelines \& biomarker assay performance (Part 3 - LBA: immunogenicity, biomarkers and PK assays). Bioanalysis 9(24), 1967-1996 (2017).

19 US Department of Health and Human Services, US FDA, Center for Drug Evaluation and Research, Center for Veterinary Medicine. Draft Guidance for Industry, Bioanalytical Method Validation. Rockville, MD, USA (2013).

20 European Medicines Agency. Committee for Medicinal Products for Human Use (CHMP). Guideline on bioanalytical method validation. London, UK (2011). EMEA/CHMP/EWP/192217/2009.

21 Japanese Ministry of Health, Labour and Welfare. Guideline on bioanalytical method validation in pharmaceutical development. Japan (2013). http://www.nihs.go.jp/drug/BMV/250913_BMV-GL_E.pdf

22 US FDA. Guidance for industry: safety testing of drug metabolites. www.fda.gov/downloads/Drugs/GuidanceComplianceRegulatoryInformation/Guidances/ucm079266.pdf

23 Piccoli SP; Neoteric Consulting \& John Michael Sauer, Critical Path Institute. Points to consider document: scientific and regulatory considerations for the analytical validation of assays used in the qualification of biomarkers in biological matrices. https://healthpolicy.duke.edu/sites/default/files/atoms/files/cpath_ptc_biomarker_qualification_assays_20170623.pdf

24 European Medicines Agency; Committee for Medicinal Products for Human Use (CHMP). Questions \& answers: positions on specific questions addressed to the Pharmacokinetics Working Party (PKWP). London, UK. EMA/618604/2008 Rev. 13 (2015).

25 Lowes S, Di Donato L, Karemud J et al. Recommendations on: internal standard criteria, stability, incurred sample reanalysis and recent 483s by the Global CRO Council for Bioanalysis. Bioanalysis 3(12), (2011).

26 Garofolo W, Savoie N. The Decennial Index of the White Papers in Bioanalysis: 'a decade of recommendations (2007-2016)'. Bioanalysis 9(21), 1681-1704 (2017). 
27 ICH final endorsed concept paper M10: bioanalytical method validation (October 2016). http://www.ich.org/fileadmin/Public_Web_Sit e/ICH_Products/Guidelines/Multidisciplinary/M10/ICH_M10_Concept_paper_final_7Oct2016.pdf

28 US Department of Health and Human Services, US FDA, Center for Drug Evaluation and Research, Center for Veterinary Medicine. Guidance for Industry, Bioanalytical Method Validation. Rockville, MD, USA (2018).

29 ICH M3(R2). Guidance for industry: nonclinical safety studies for the conduct of human clinical trials and marketing authorization for pharmaceuticals (2010). http://www.fda.gov/downloads/drugs/guidances/ucm073246.pdf

30 ICH M3(R2). Questions and answers (R2) on nonclinical safety studies for the conduct of human clinical trials and marketing authorization for pharmaceuticals (2013).

http://www.fda.gov/downloads/drugs/guidancecomplianceregulatoryinformation/guidances/ucm292340.pdf

31 Gao H, Obach RS. Addressing MIST (Metabolites in Safety Testing): bioanalytical approaches to address metabolite exposures in humans and animals. Curr. Drug Metab. 12(6), 578-586 (2011).

32 Schadt S, Bister B, Chowdhury SK et al. A decade in the MIST: learnings from investigations of drug metabolites in drug development under the 'Metabolites in Safety Testing' regulatory guidances. Drug Metab. Dispos. 46(6), 856-878 (2018).

33 ICH S9. Nonclinical evaluation for anticancer pharmaceuticals for the safety testing of drug metabolites in cancer therapies. https://www.fda.gov/downloads/Drugs/Guidances/ucm085389.pdf

34 Jones BR, Schultz GA, Eckstein JA, Ackermann BL. Surrogate matrix and surrogate analyte approaches for definitive quantitation of endogenous biomolecules. Bioanalysis 4(19), 2343-2356 (2012).

35 Houghton R, Gouty D, Allinson J et al. Recommendations on biomarker bioanalytical method validation by GCC. Bioanalysis 4(20), 2439-2446 (2012).

36 Cape S, Isla R, Nehls C et al. The 10th GCC Closed Forum: rejected data, GCP in bioanalysis, extract stability, BAV, processed batch acceptance, matrix stability, critical reagents, ELN and data integrity and counteracting fraud. Bioanalysis 9(7), 505-516 (2017).

37 Arnold ME, Booth B, King L, Ray C. Workshop report: Crystal City VI - bioanalytical method validation for biomarkers. AAPS J. 18(6), 1366-1372 (2016).

38 Zheng K, Ji A, Chung L et al. Enhancement of human plasma glucosylceramide assay sensitivity using delipidized plasma. Mol. Genet. Metab. Reports 8, 77-79 (2016).

39 Luo L, Aubrecht J, Li D et al. Assessment of serum bile acid profiles as biomarkers of liver injury and liver disease in humans. PLOS ONE 13(3), e0193824 (2018).

40 International Transporter Consortium; Giacomini KM, Huang SM et al. Membrane transporters in drug development. Nat. Rev. Drug Discov. 9(3), 215-236 (2010).

41 Takehara I, Terashima H, Nakayama T et al. Investigation of glycochenodeoxycholate sulfate and chenodeoxycholate glucuronide as surrogate endogenous probes for drug interaction studies of OATP1B1 and OATP1B3 in healthy Japanese volunteers. Pharm. Res. 34(8), 1601-1614 (2017).

42 Lai Y, Mandlekar S, Shen H et al. Coproporphyrins in plasma and urine can be appropriate clinical biomarkers to recapitulate drug-drug interactions mediated by organic anion transporting polypeptide inhibition. J. Pharmacol. Exp. Ther. 358(3), 397-404 (2016).

43 Müller F, Pontones CA, Renner B et al. N1-methylnicotinamide as an endogenous probe for drug interactions by renal cation transporters: studies on the metformin-trimethoprim interaction. Eur. J. Clin. Pharmacol. 71(1), 85-94 (2015).

44 Ito $\mathrm{S}$, Kusuhara $\mathrm{H}$, Kumagai $\mathrm{Y}$ et al. $\mathrm{N}$-methylnicotinamide is an endogenous probe for evaluation of drug-drug interactions involving multidrug and toxin extrusions (MATE1 and MATE2-K). Clin. Pharmacol. Ther. 92(5), 635-41 (2012).

45 Ramanathan R, King-Ahmad AJ, Holliman CL, Rodrigues AD. A highly selective and sensitive LC-MS/HRMS assay for quantifying coproporphyrins as organic anion-transporting peptide biomarkers. Bioanalysis 9(22), 1787-1806 (2017).

46 Njumbe Ediage E, Dillen L, Vroman A et al. Development of an LC-MS method to quantify coproporphyrin I and III as endogenous biomarkers for drug transporter-mediated drug-drug interactions. J. Chromatogr. B Analyt. Technol. Biomed Life Sci. 1073, 80-89 (2018).

47 King-Ahmad A, Clemens S, Ramanathan R et al. A fully automated and validated human plasma LC-MS/MS assay for endogenous OATP biomarkers coproporphyrin-I and coproporphyrin-III. Bioanalysis 10(9), 691-701 (2018).

48 Kandoussi H, Zeng J, Shah K et al. UHPLC-MS/MS bioanalysis of human plasma coproporphyrins as potential biomarkers for organic anion-transporting polypeptide-mediated drug interactions. Bioanalysis 10(9), 633-644 (2018).

49 Kunze A, Ediage EN, Dillen L et al. Clinical investigation of coproporphyrins as sensitive biomarkers to predict mild to strong OATP1B-mediated drug-drug interactions. Clin Pharmacokinet. 57(12), 1559-1570 (2018).

50 Rago B, Tierney B, Rodrigues AD et al. A multiplex HRMS assay for quantifying selected human plasma bile acids as candidate OATP biomarkers. Bioanalysis 10(9), 645-657 (2018).

51 Luo L, Kay J, Zhang J et al. LC-MS/MS assay for N(1)-methylnicotinamide in humans, an endogenous probe for renal transporters. Bioanalysis 10(9), 673-689 (2018).

52 Tremblay G, Oldfield P. Bioanalysis of siRNA and oligonucleotide therapeutics in biological fluids and tissues. Bioanalysis 1(3), 595-609 (2009). 
53 Collins ML, Irvine B, Tyner D et al. A branched DNA signal amplification assay for quantification of nucleic acid targets below 100 molecules/ml. Nucleic Acids Res. 25(15), 2979-2984 (1997).

54 Laixin Wang, Chengjie Ji. Advances in quantitative bioanalysis of oligonucleotide biomarkers and therapeutics. Bioanalysis 8(2), 143-155 (2016).

55 Wang Z, Yang B. End-point stem-loop real-time RT-PCR for miRNA quantification. In: MicroRNA Expression Detection Methods. Springer, Berlin, Heidelberg, Germany, 131-140 (2010).

56 Ro S, Park C, Jin J, Sanders KM, Yan W. A PCR-based method for detection and quantification of small RNAs. Biochem. Biophys. Res. Commun. 351(3), 756-763 (2006).

57 European Medicines Agency. ICH considerations: general principles to address virus and vector shedding. London, UK. EMEA/CHMP/ICH/449035/2009 (2009).

58 European Medicines Agency. EMA draft guideline on the quality, non-clinical and clinical aspects of gene therapy medicinal products. London, UK. EMA/CAT/80183/2014 (2015).

59 US Department of Health and Human Services, US FDA, Center for Biologics Evaluation and Research. Guidance for Industry, Design and Analysis of Shedding Studies for Virus or Bacteria-Based Gene Therapy and Oncolytic Products. Silver Spring, MD, USA (2015).

60 Sahin U, Karikó K, Türeci O. mRNA-based therapeutics - developing a new class of drugs. Nat. Rev. Drug Discov. 13, 759-780 (2014).

61 Ligon TS, Leonhardt C, Rädler JO. Multi-level kinetic model of mRNA delivery via transfection of lipoplexes. PLoS ONE 9(9), e107148 (2014).

62 Coles AH, Osborn MF, Alterman JF et al. A high-throughput method for direct detection of therapeutic oligonucleotide-induced gene silencing in vivo. Nucleic Acid Ther. 26(2), 86-92 (2016).

63 Sedic M, Joseph J, Lynn A et al. Safety evaluation of lipid nano-particles-formulated modified mRNA in the Sprague-Dawley rat and cynomolgus monkey. Vet Pathol. 55(2), 341-354 (2018).

64 Ren S, Fier PS, Ren H et al. ${ }^{34}$ S: a new opportunity for the efficient synthesis of stable isotope labeled compounds. Chem. Eur. J. 24(28), 7069-7272 (2018).

65 FDA-NIH Biomarker Working Group. BEST (Biomarkers, EndpointS, and other Tools) resource.. Silver Spring, US FDA; Bethesda and NIH, MD, USA (2016).

66 Piccoli S, Sauer JM, Amur S et al.Public workshop: scientific and regulatory considerations for the analytical validation of assays used in the qualification of biomarkers in biological matrices. https:

//healthpolicy.duke.edu/events/public-workshop-scientific-and-regulatory-considerations-analytical-validation-assays-used

\section{Affiliations}

${ }^{1}$ EU EMA, London, UK

${ }^{2}$ Alnylam Pharmaceuticals, Cambridge, MA, USA

${ }^{3}$ GlaxoSmithKline, King of Prussia, PA, USA

${ }^{4}$ AstraZeneca, Cambridge, UK

${ }^{5}$ AstraZeneca, Gothenburg, Sweden

${ }^{6}$ Pfizer, Groton, CT, USA

${ }^{7}$ Bristol-Myers Squibb, Princeton, NJ, USA

${ }^{8}$ Pharmascience, Montreal, QC, Canada

${ }^{9}$ Amgen Research, Thousand Oaks, CA, USA

${ }^{10} \mathrm{~F}$ Hoffmann-La Roche Ltd, Basel, Switzerland

${ }^{11}$ Merck Research Labs, West Point, PA, USA

${ }^{12}$ GlaxoSmithKline, Ware, UK

${ }^{13}$ Janssen Research \& Development, Beerse, Belgium

${ }^{14}$ Biopharma Services, Toronto, ON, Canada

${ }^{15}$ PPD, Richmond, VA, USA

${ }^{16}$ Moderna Therapeutics, Cambridge, MA, USA

${ }^{17}$ Intertek, San Diego, CA, USA

${ }^{18}$ Health Canada, Ottawa, ON, CAN

${ }^{19}$ US FDA, Silver Spring, MD, USA

${ }^{20}$ UK MHRA-NIBSC, London, UK

${ }^{21}$ Princeton, NJ, USA

\author{
${ }^{22}$ Angelini Pharma, Pomezia, RM, Italy \\ ${ }^{23}$ LGC, Cambridge, UK \\ ${ }^{24}$ Japan MHLW-NIHS, Tokyo, Japan \\ ${ }^{25}$ Wave Life Sciences, Cambridge, MA, USA \\ ${ }^{26}$ Sanofi, Framingham, MA, USA \\ ${ }^{27}$ Q2 Solutions, Ithaca, NY, USA \\ ${ }^{28}$ Alexion Pharmaceuticals, New Haven, CT, USA \\ ${ }^{29}$ Stock Therapeutics, Bedford, MA, USA \\ ${ }^{30}$ Brazil Anvisa, Brasilia, Brazil \\ ${ }^{31}$ Ionis Pharmaceuticals, Carlsbad, CA, USA \\ ${ }^{32}$ Regulus, San Diego, CA, USA \\ ${ }^{33}$ Neoteric, Princeton, NJ, USA \\ ${ }^{34}$ LGC Axolabs GmbH, Kulmbach, Germany \\ ${ }^{35}$ Medlmmune LLC, South San Francisco, CA, USA \\ ${ }^{36}$ Charles River Labs, Edinburgh, UK \\ ${ }^{37}$ CFABS, Montreal, QC, CAN \\ ${ }^{38}$ Biogen, Cambridge, MA, USA \\ ${ }^{39}$ AbbVie Inc., North Chicago, IL, USA \\ ${ }^{40}$ PRA Health Sciences, Assen, The Netherlands \\ ${ }^{41}$ UK MHRA, London, UK
}

Disclaimer: The views expressed in this article are those of the authors and do not reflect official policy of the US FDA, Europe EMA, UK MHRA, Brazil ANVISA, Health Canada, Japan MHLW, and Finland Fimea. No official endorsement by the FDA, EMA, Health Canada, MHRA, ANVISA, MHLW or Fimea is intended or should be inferred. 
